Supporting Information for

\title{
Substrate-Induced Nanoscale Undulations of Borophene on Silver
}

\author{
Zhuhua Zhang ${ }^{1}$, Andrew J. Mannix ${ }^{2,3}$, Zhili $\mathrm{Hu}^{1}$, Brian Kiraly ${ }^{2,3}$, Nathan P. Guisinger ${ }^{2}$, Mark C. \\ Hersam $^{3,4}$, and Boris I. Yakobson ${ }^{1}$ \\ ${ }^{1}$ Department of Materials Science and NanoEngineering and Department of Chemistry, Rice \\ University, Houston, TX 77005, USA \\ ${ }^{2}$ Center for Nanoscale Materials, Argonne National Laboratory, 9700 South Cass Avenue, \\ Building 440, Argonne, IL 60439, USA \\ ${ }^{3}$ Department of Materials Science and Engineering, Northwestern University, 2220 Campus \\ Drive, Evanston, IL 60208, USA \\ ${ }^{4}$ Department of Chemistry, Northwestern University, 2220 Campus Drive, Evanston, IL 60208, \\ USA
}

First-principles calculations: The calculations are performed by employing ultrasoft pseudopotentials for the core region and spin-unpolarized density functional theory (DFT) based on the generalized gradient approximation of Perdew-Burke-Ernzerhof functional, as implemented in Vienna Ab-initio Simulation Package (VASP) code. A kinetic energy cutoff of $400 \mathrm{eV}$ is chosen for the plane-wave expansion. In all structures, the vacuum region between two adjacent periodic images is fixed to $12 \AA$ to eliminate spurious interaction. The Brillouin-zone integration is densely sampled according to the supercell size, ensuring approximately the same $\boldsymbol{k}$-point density among different-sized supercells. The model systems consist of a B sheet on flat or reconstructed $\operatorname{Ag}(111)$ consisting of four atomic layers (steps not included). For the undulated 2D B on reconstructed substrate, we use a $1 \times 14$ supercell for the substrate to match a $1 \times 15$ supercell for the $v_{1 / 6}$ sheet, whereas for the flat 2D B on flat $\operatorname{Ag}(111)$, we use a $1 \times 1$ unit cell for the $\mathrm{v}_{1 / 6}$ sheet and a $1 \times \sqrt{3}$ supercell for the substrate. The partially undulated $\mathrm{B}$ sheets are simulated by using sufficiently wide B nanoribbons with H-terminated edges, which are then placed on a partially reconstructed substrate with a lateral dimension up to $100 \AA$; the nearest atomic distance between two nanoribbons in adjacent images is kept larger than $8 \AA$ to avoid interaction. The positions of metal atoms of the topmost three layers plus the step Ag atoms and the entire B sheet are fully relaxed using the conjugate-gradient method until the force on each atom is less than $0.01 \mathrm{eV} / \AA$. Dipole correction are included in calculating the total energies. Scanning tunneling microscopy (STM) images are simulated in constant current mode and rendered with Hive software.

Cluster expansion method: The $2 \mathrm{D}$ B sheet can be viewed as a triangular lattice (B) with a pattern of hexagon vacancies (denoted as []) and treated as an alloy $\mathrm{B}_{1-\mathrm{v}}[]_{\mathrm{v}}$; The rhombic $2 \times 2$ and rectangular $2 \times 2 \sqrt{3}$ supercells are employed respectively as the initial unit cell, and then larger supercells, up to $42 \mathrm{~B}$ atoms, are screened. The generated structure from the unit cells can be described by a vector $\boldsymbol{\sigma}=\left\{\sigma_{1}, \sigma_{2}, \ldots, \sigma_{n}\right\}$, with $\sigma_{i}=+1$ for the hexagon center where a B atom is present and -1 if not. The total energy $E$ for a particular configuration $\boldsymbol{\sigma}$ can be expanded as series $E(\boldsymbol{\sigma})=J_{0}+(1-2 \eta) J_{1}+\sum d_{\mathrm{x}} J_{\mathrm{x}} \Pi_{\mathrm{x}}(\boldsymbol{\sigma})$, where $d_{x}$ is the number of clusters of type $x$, which can be pairs, triplets, etc., $J_{\alpha}$ is respective effective cluster interaction, and $\Pi_{x}(\boldsymbol{\sigma})=\left\langle\Pi_{i} \sigma_{i}\right\rangle$ represents 
the "spin" products for configuration $\boldsymbol{\sigma}$ averaged over all symmetry-equivalent $x$-type clusters. The expansion coefficients $J_{x}$ are determined by fitting the energies to the direct DFT-computed values of generated structures, as implemented in ATAT (alloy theoretical automated toolkit) code. After the structural research, we further select 20 structures with the lowest energies for higher-precision optimization and energy calculations. The relative positions of these B sheets on substrates are further checked to ensure the structures reach the optimal registry.

\section{Growth method and characterizations:}

Our 2D boron sheets are grown on single-crystal $\mathrm{Ag}(111)$ substrates in ultra-high vacuum, which were cleaned via repeated cycles of Ar sputtering followed by annealing at $\sim 820 \mathrm{~K}$. During the course of growth, boron was deposited from an electron beam evaporator (Focus Gmbh.) with a boron rod source (ESPI metals, 99.9999\%). The deposition rate was set to vary from 0.01 to 0.1 $\mathrm{ML} / \mathrm{min}$. Other details on the growth can be found in our previous work. To control the undulation coverage in the B sheets, the samples were heated to a temperature varying from 720 $\mathrm{K}$ to $980 \mathrm{~K}$, by a button heater. Then, the samples grown at different temperatures were cooled down and STM measurements were conducted in an Omicron Nanotechnology VT-SPM at $55 \mathrm{~K}$ for in-situ characterization. STM measurements were acquired in constant current mode, using electrochemically etched W tips degassed in situ. In order to reduce apparent noise in some STM images, a Gaussian filter was applied with radii of 2-3 pixels. 


\section{Supporting discussions}

Critical strain: for a 2D boron sheet under infinitesimal strain, we have $D \Delta^{2} w+N_{x} \Delta w+c w=0$,

where $D$ is the bending stiffness, $N_{\mathrm{x}}=C \varepsilon$ is the compressive force in the x direction, $C$ is the inplane stiffness, and $c$ is the spring constant. With $w=2 A \sin (\omega x)$, eq. $\mathrm{S} 1$ becomes

$D \alpha^{2}-N_{x} \alpha+c=0$,

Obviously, to avoid non-imaginary solution in eq. S2, $N_{x}$ must satisfy

$N_{x}=C \varepsilon \geq 2 \sqrt{ } D c$,

Therefore, the critical strain for the appearance of undulation is $\varepsilon_{\mathrm{cr}}=2 \sqrt{D c} / C$. With $C=189 \mathrm{~N} / \mathrm{m}$, $D=0.39 \mathrm{eV}$, and $c=0.032 \mathrm{eV} / \AA^{4}$ from DFT calculations, $\varepsilon_{\mathrm{cr}}$ is determined to be $1.9 \%$.

Optimal wave length: We apply an out-of-plane perturbation to the compressed plate as $w=$ $h \sin (\omega x) / 2$, where $h$ is the height of undulation and $\omega=2 \pi / \lambda$ is the circular frequency. Since $\partial_{y} w$ $=0$, the free energy per period of the system can be expressed as:

$F=\int_{S}\left\{\frac{C}{2}\left[\varepsilon_{0}-\left(\int_{0}^{\lambda} \frac{1}{\lambda} \sqrt{1+w_{x}^{2}} d x-1\right)^{2}\right]^{2}+\frac{D}{2} w_{x x}{ }^{2}+p(w)\right\} \mathrm{d} x y$,

where $\varepsilon_{0}$ is the pre-strain, $S$ is the surface area of boron sheet and $p(w)$ is the off substrate potential. Note that the Poisson ratio of plate is omitted here. When $w<<\lambda, \sqrt{1+w_{x}^{2}} \approx 1+w_{x}^{2} / 2$. Thus eq. S4 becomes.

$F=\int_{S}\left\{\frac{C}{2}\left[\varepsilon_{0}-\left(\int_{0}^{\lambda} \frac{1}{2} w_{x}^{2} / d x\right)^{2}\right]^{2}+\frac{D}{2} w_{x x}^{2}+p(w)\right\} \mathrm{d} x y$,

We use a harmonic approximation to describe $p$ as $p(w)=c w^{2} / 2$, where $c$ is the spring constant. eq. S5 can be further expressed as:

$F / S=\frac{C}{2}\left[\varepsilon_{0}-(h \pi / \lambda)^{2} / 4\right]^{2}+D\left(h \pi^{2} / \lambda^{2}\right)^{2}+\frac{c}{16} h^{2}$,

To get the analytic expression of $\lambda$, we further presume the pre-strain is fully released, i.e.

$\boldsymbol{\varepsilon}_{0}=(h \boldsymbol{\pi} / \boldsymbol{\lambda})^{2} / 4$. With this, eq. S6 can be simplified as

$F / S=4 D \pi^{2} \varepsilon_{0} / \lambda^{2}+\frac{c \varepsilon_{0} \lambda^{2}}{4 \pi^{2}}$.

Minimizing eq. S7 vs $\lambda$ results in $\lambda=2 \pi(D / c)^{0.25}$. 


\section{Geometric analyses:}

If the in-plane strain is fully relaxed in the undulated 2D B sheet, its total length should be conserved from a flat B sheet. Thus, we have

$\frac{\lambda}{1-\varepsilon}=\int_{-\pi}^{\pi} \sqrt{\left(1+h^{2} w^{2} \sin ^{2} w x\right)} d x$

where $h$ is the height of undulation, $\lambda$ is the wave length and $\varepsilon$ is the pre-strain.

According to eq. S8, we have

$\lambda /(1-\varepsilon) \approx \sqrt{\lambda^{2}+\pi^{2} h^{2}}\left(1-\pi^{2} h^{2} / 4\left(\lambda^{2}+\pi^{2} h^{2}\right)\right)$,

Let $\mathrm{a}=h / \lambda$, eq. $\mathrm{S} 9$ can be written as

$\varepsilon \approx 1-\frac{4\left(1+\pi^{2} \alpha^{2}\right)}{\sqrt{1+\pi^{2} \alpha^{2}}\left(4+3 \pi^{2} \alpha^{2}\right)}$.

eq. S10 is numerically illustrated in the Figure below.

a
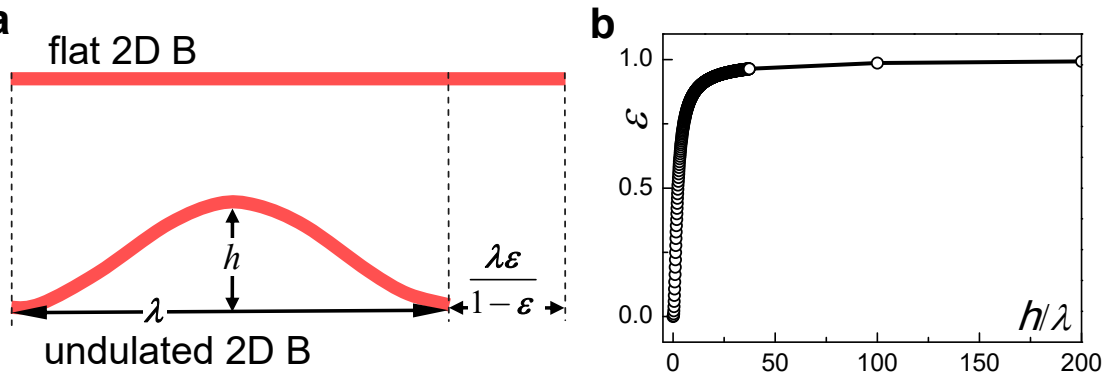

Figure (a) Schematic illustration of the length of a flat and an undulated 2D B sheets. (b) A numerical solution of the pre-strain $\varepsilon$ as a function of $a(=h / \lambda)$. 

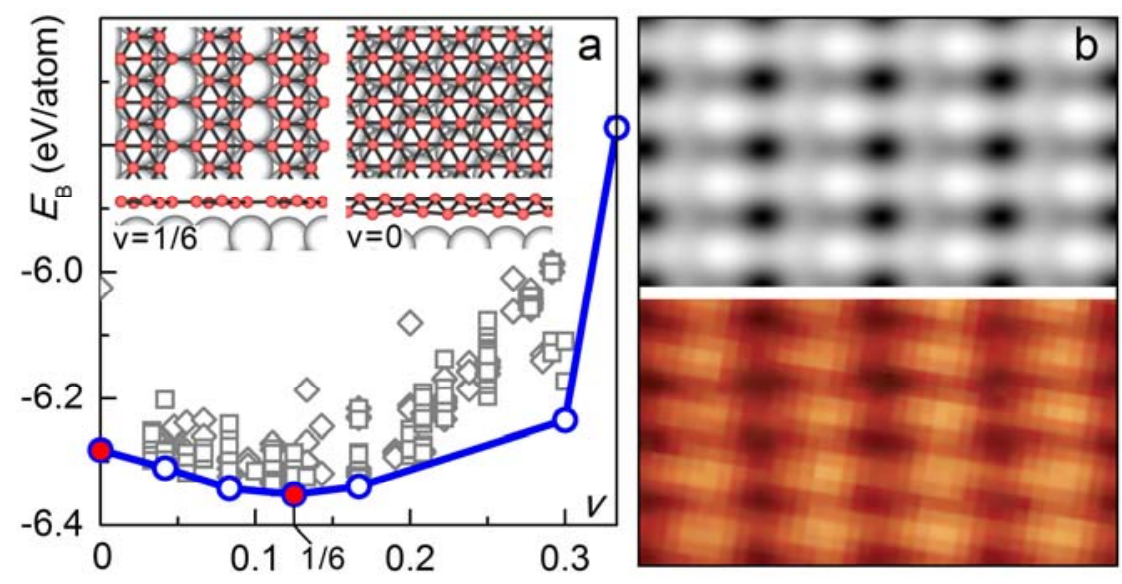

Figure S1. Structural search of 2D B on Ag(111). (a) Total energy per B of all B sheets calculated by cluster-expansion-fits on $\mathrm{Ag}$ as a function of vacancy concentration $v$. Inset: top and side views of the low-energy monolayer structures at $\mathrm{v}=0$ and $1 / 6$, respectively. (b) Simulated STM image (top) at $V_{\text {sample }}=1.0 \mathrm{~V}$ for the $\mathrm{v}_{1 / 6}$ sheet, together with an experimental STM image (bottom) at $V_{\text {sample }}=0.1 \mathrm{~V}$ and $I_{\mathrm{t}}=1.0 \mathrm{nA}$.

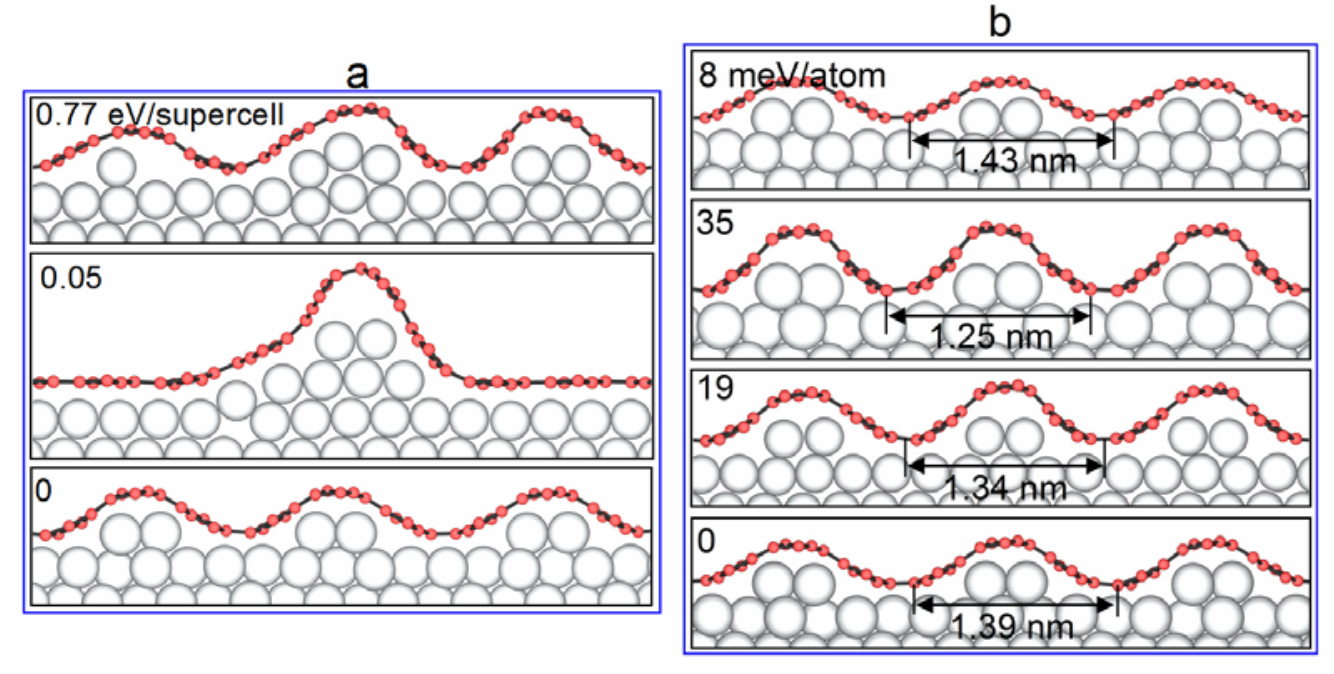

Figure S2. Energy comparison of differently undulated borophenes. (a) Total energy per supercell for the undulated $\mathrm{v}_{1 / 6}$ sheet on three reconstructed $\operatorname{Ag}(111)$ surfaces with different step heights. (b) Energy per B atom for an undulated $v_{1 / 6}$ sheet on four reconstructed $\operatorname{Ag}(111)$ surfaces with different inter-step distances. The number in each panel is the energy of that configuration with respect to the optimal case shown at the bottom. 

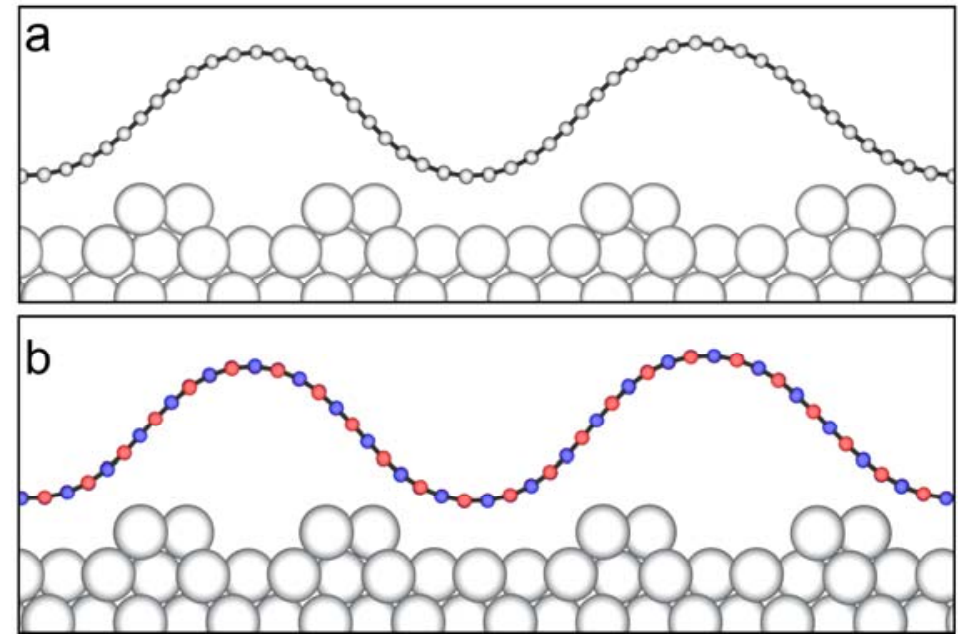

Figure S3. Graphene and h-BN on reconstructed $\operatorname{Ag}(111)$. The cases of (a) graphene and (b) h-BN sheet are shown. Note that both the undulated structures are much less stable than their planar forms on flat $\mathrm{Ag}(111)$.
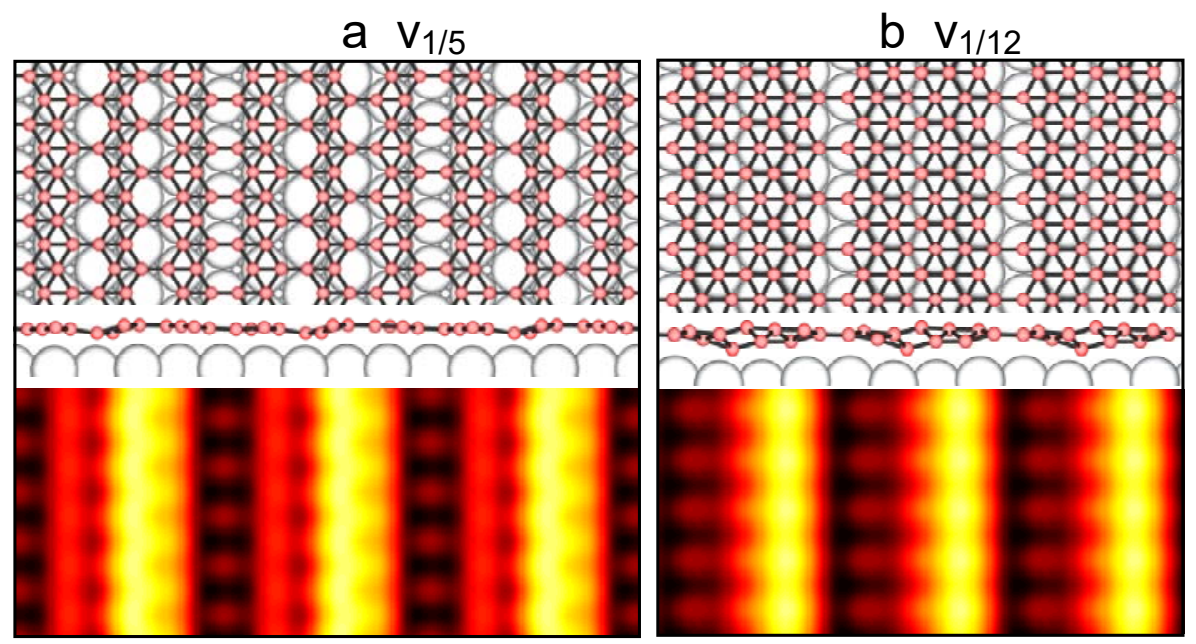

Figure S4. Simulated STM images of two planar borophenes on $\mathbf{A g ( 1 1 1 ) . ~ ( a ) ~} \mathrm{v}_{1 / 5}$ and (b) $\mathrm{v}_{1 / 12}$ sheets are shown. Top: front and side views of the B sheets on silver. Bottom: simulated STM images of the two sheets with $V_{\text {sample }}=1.0 \mathrm{~V}$. 


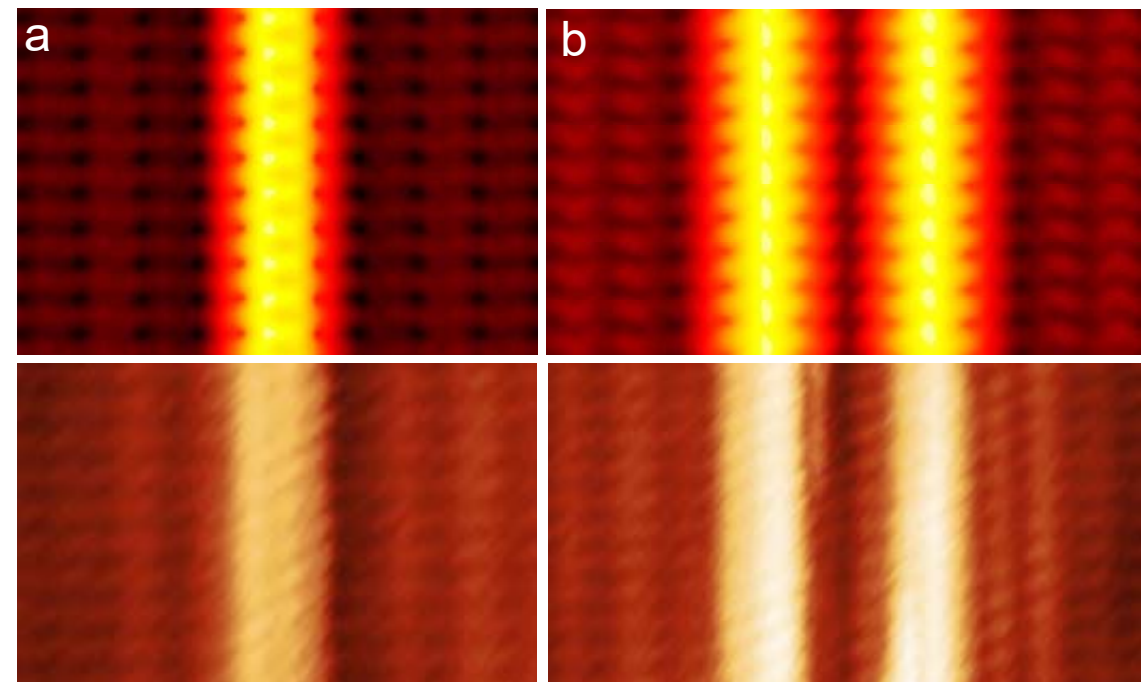

Figure S5. Simulations of partially undulated borophenes. Simulated (top) and experimental (bottom) STM images of the partially undulated sheet with (a) one and (b) two crests. $V_{\text {sample }}=$ $1.0 \mathrm{~V}$ for simulations; $V_{\text {sample }}=0.2 \mathrm{~V}, I_{\mathrm{t}}=1.0 \mathrm{nA}$ for experiments. 


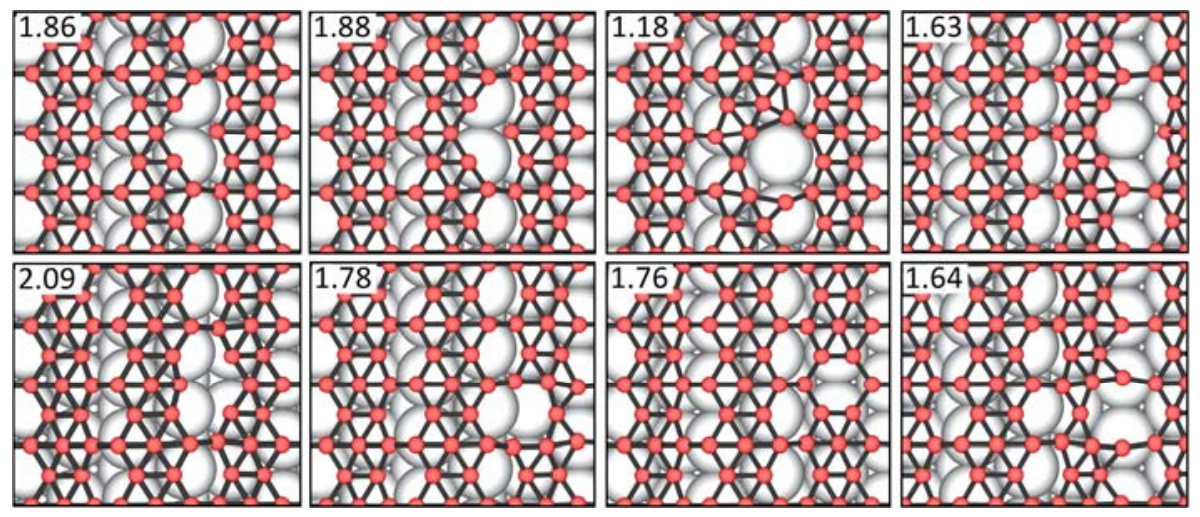

Figure S6. Monovacancy in undulated borophene. Defect structures by removing a B atom from site 1 to site 8 (see Figure 3a in text) are shown in sequence (left to right, top to bottom). The number in each panel is the defect formation energy in units of $\mathrm{eV}$.
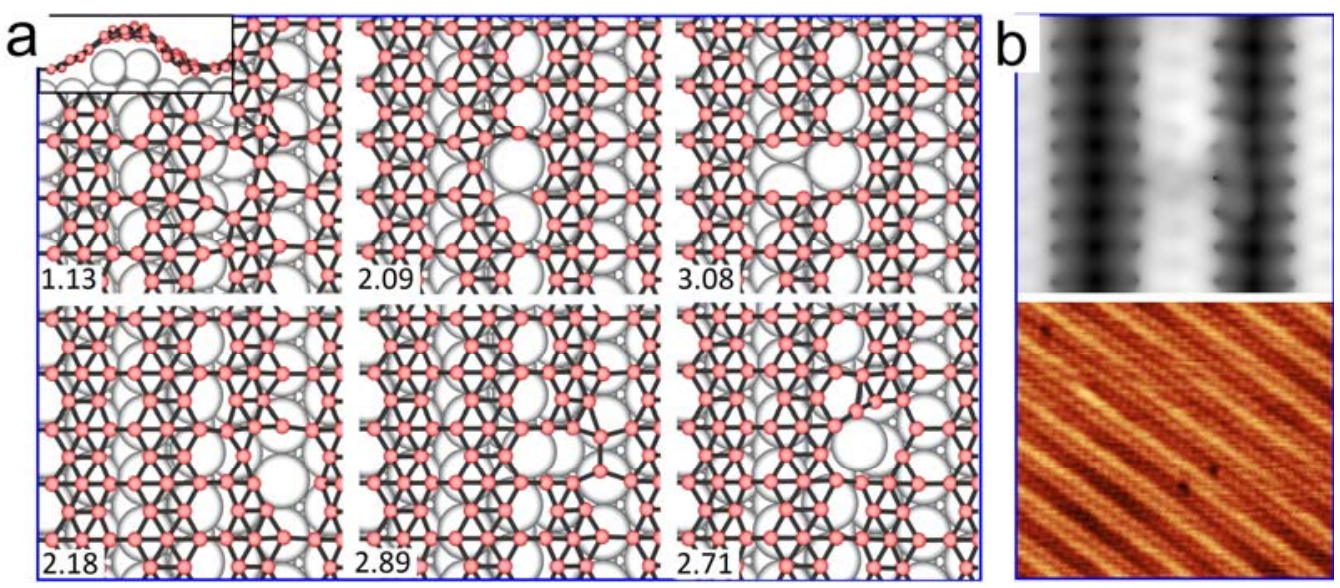

Figure S7. Double-vacancy in undulated borophene. (a) Low-energy defect structures. The number in each panel is the defect formation energy in units of eV. (b) Simulated STM image (top) of the energetically optimal double-vacancy, compared to the experimental image (bottom) of an undulated B sheet with vacancy defects. $V_{\text {sample }}=1.0 \mathrm{~V}$ for simulations; $V_{\text {sample }}=0.1 \mathrm{~V}, I_{\mathrm{t}}=$ $1.0 \mathrm{nA}$ for experiments. 

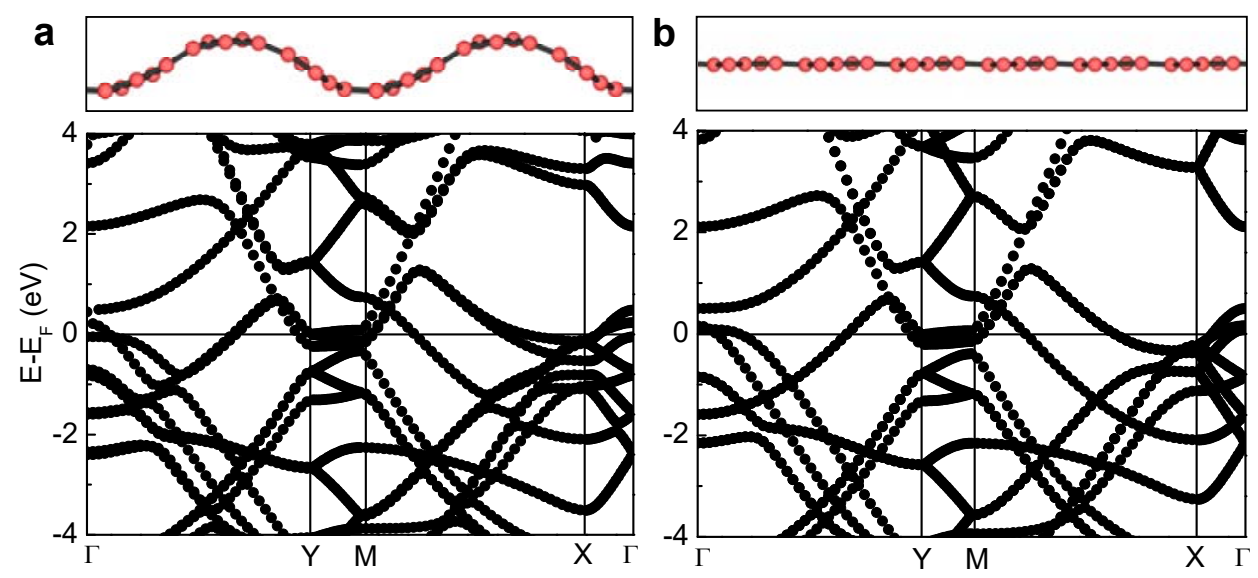

Figure S8. Electronic properties of the undulated borophene. Band structures of (a) an undulated and (b) a flat borophene in vacuum, together with the corresponding atomic structures.

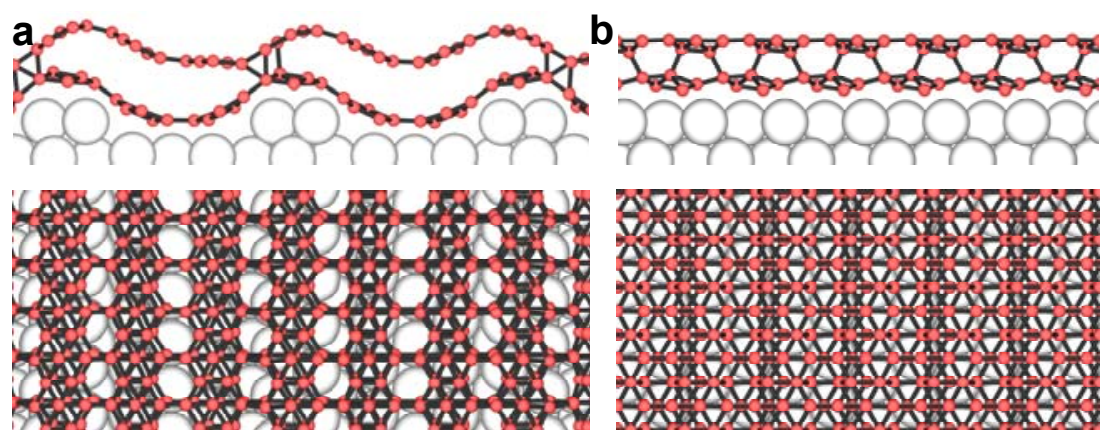

Figure S9. Relaxed atomic configuration of a bilayer (a) undulated and (b) flat $\mathrm{v}_{1 / 6}$ sheets on reconstructed and flat $\mathrm{Ag}(111)$. 